\title{
Acute massive pulmonary embolism Clinical and haemodynamic findings in 23 patients studied by cardiac catheterization and pulmonary arteriography
}

\author{
G. A. H. Miller ${ }^{1}$ and G. C. Sutton ${ }^{2}$ \\ From the Cardiac Department, Brompton Hospital, London S.W.3
}

Twenty-three patients are reported in whom a diagnosis of acute massive pulmonary embolism was confirmed by pulmonary arteriography. All patients had a history of less than 48 hours' duration and only two had previous cardiorespiratory disease. In such patients the haemodynamic abnormalities determined at catheterization are due to pulmonary embolism as an isolated disturbance. These abnormalities include an only moderate degree of pulmonary hypertension (PA systolic pressure $38.4 \pm 6.8 \mathrm{~mm}$. $\mathrm{Hg}$ ), right ventricular 'failure' (RVED II.5 $\pm 4.9 \mathrm{~mm}$. $\mathrm{Hg}$ ), arterial oxygen desaturation $(86 \cdot 4 \pm I I \cdot 2 \%)$ and a wide arteriovenous oxygen difference $(8 \cdot I \pm$ $I .8 \mathrm{ml} . / 100 \mathrm{ml}$.), and low cardiac output. These haemodynamic abnormalities find their expression in the presentation and the clinical, electrocardiographic, and radiological findings which are described.

The clinical presentation of pulmonary embolism and the haemodynamic disturbance caused by such embolism may be modified by the size of the embolus, its duration, and the presence or absence of pre-existing cardiorespiratory disease. The purpose of the present communication is to describe the clinical and haemodynamic findings in a homogeneous group of 23 patients all of whom were shown by pulmonary arteriography to have suffered massive pulmonary embolism and all of whom were studied within 48 hours of the acute event; in only 2 patients was there any preexisting cardiorespiratory disease. Such patients provide a unique opportunity for studying, in man, the effects of acute right ventricular outflow obstruction in a previously normal cardiorespiratory system.

\section{Selection of patients}

The 23 patients reported ( 9 male, 14 female, age range I4-64 years) represent all patients referred over a 3-year period in whom a diagnosis of massive pulmonary embolism was confirmed by pulmonary arteriography. Seventeen patients Received 6 February 1970.

1 Correspondence and requests for reprints to Dr. G. A. H. Miller.

2 This study forms part of an MD thesis submitted to the University of Cambridge. were seen and investigated within 24 hours of the acute event ( 7 at less than 6 hours) and 6 patients between 24 and 48 hours. In 16 of these patients, most of whom sustained massive pulmonary embolism after a surgical procedure, the onset of massive embolism was indicated by the occurrence of collapse which often required resuscitative measures. In 7 patients timing of the acute episode, and, therefore, the duration of embolism, was more difficult - particularly when there had been premonitory episodes of pulmonary infarction. In these patients massive embolism was presumed to have occurred at the time of a sudden deterioration in the clinical state of the patient. In all patients pulmonary arteriography showed emboli involving at least half of the major pulmonary arterial branches. The extent of embolization was confirmed by pulmonary embolectomy in 12 of these patients. The remaining II patients were treated with streptokinase. ${ }^{1}$ There was no mortality in either group. Only 2 patients had pre-existing cardiorespiratory disease; one (Case 14) had a myocardial infarct 3 weeks before sustaining massive pulmonary embolism, while the other (Case 6) had mild unsuspected mitral stenosis.

\section{Method of investigation}

After clinical assessment, including a plain chest $x$-ray and standard 12 lead electrocardiogram, all

1 Kabikinase, Kabi Pharmaceuticals Ltd., Bilton House, Uxbridge Road, London W.5. 
patients were studied by right heart catheterization using conventional techniques, pressure measurements being referred to the mid-chest level. Cardiac index) $1 . / \mathrm{min}$./sq.m.) was calculated by the Fick principle, expired air being collected and analysed for oxygen and carbon dioxide content by the micro-Scholander technique (Scholander, 1947). In most patients, however, the clinical condition did not permit collection of an expired air sample, and cardiac output was calculated using an assumed oxygen consumption from the data of Robertson and Reid (I952). Body surface area was determined from nomograms constructed according to the formula of Du Bois and $\mathrm{Du}$ Bois (1916). Samples were obtained simultaneously from the pulmonary and brachial artery and analysed for oxygen saturation using a haemoreflector (Zijlstra and Mook, I962). Antero-posterior pulmonary arteriograms were obtained using $\mathrm{I} \mathrm{ml} . / \mathrm{kg}$. body weight of 75 per cent Triosil $^{1}$ injected into the main pulmonary artery. Rapid serial exposures were obtained using an Elema Schönander roll film changer.

\section{Results}

(I) Clinical findings The clinical findings

${ }_{1}$ Sodium, calcium, and magnesium metrizoate. Glaxo Laboratories Ltd., Greenford, Middlesex. and haemodynamic findings are given in the Table.

\section{(a) Presentation}

The acute incident of massive pulmonary embolism was characterized by the sudden onset of dyspnoea (20 of 23 patients-87\%) and 'collapse' $(70 \%)$. Five patients $(22 \%)$ developed central chest pain simulating myocardial infarction. Circulatory arrest requiring external cardiac massage occurred in 3 patients, and was more commonly seen in a larger group of patients reported elsewhere (Sutton, Honey, and Gibson, 1969) with acute massive pulmonary embolism, not all of whom were investigated by cardiac catheterization. In II patients $(48 \%)$ there was a history suggesting an episode of minor pulmonary embolism preceding the massive episode by up to 7 days. These earlier episodes were usually associated with pleurisy or haemoptysis and suggestive alterations consistent with pulmonary infarction on the plain chest $x$-ray. Pleurisy was a feature in only 5 patients at the time of massive embolism and haemoptysis in only 2.

TABLE Clinical and haemodynamic findings in patients with acute massive pulmonary embolism

\begin{tabular}{|c|c|c|c|c|c|c|c|c|c|c|c|c|}
\hline \multirow{3}{*}{$\begin{array}{l}\text { Case } \\
\text { No. }\end{array}$} & \multirow{3}{*}{$\begin{array}{l}\text { Dura- } \\
\text { tion } \\
\text { (hr.) }\end{array}$} & \multirow{2}{*}{\multicolumn{2}{|c|}{$\begin{array}{l}R A \text { pressure } \\
(\mathrm{mm} . \mathrm{Hg})\end{array}$}} & \multirow{3}{*}{$\begin{array}{l}R V E D P \\
(\mathrm{~mm} . \mathrm{Hg})\end{array}$} & \multirow{2}{*}{\multicolumn{2}{|c|}{$\begin{array}{l}P A \text { pressure } \\
(\mathrm{mm} . \mathrm{Hg})\end{array}$}} & \multirow{3}{*}{$\begin{array}{c}\text { Art. } \mathrm{O}_{2} \\
\text { satn. }(\%)\end{array}$} & \multirow{3}{*}{$\begin{array}{l}A V \mathrm{O}_{2} \text { diff. } \\
\text { (ml./100 ml.) }\end{array}$} & \multirow[t]{3}{*}{ Gallop } & \multirow{3}{*}{$\begin{array}{l}\text { Second } \\
\text { heart } \\
\text { sound }\end{array}$} & \multicolumn{2}{|c|}{ Electrocardiogram ${ }^{\star}$} \\
\hline & & & & & & & & & & & Rhythm & Pattern \\
\hline & & Mean & ' $a$ ' wave & & Systolic & Mean & & & & & & \\
\hline $\mathbf{I}$ & 2 & 9 & 16 & I4 & 50 & 35 & 74 & $5 \cdot 6$ & + & Wide & ST & RBBB \\
\hline 2 & 4 & 9 & 12 & 10 & 40 & 28 & 91 & $12 \cdot 2$ & + & Single & ST & RBBB \\
\hline 3 & 5 & 8 & 9 & 12 & 34 & 25 & 94 & 10.9 & + & Single & ST & RBBB \\
\hline 4 & 5 & II & 12 & 13 & 50 & 33 & 94 & 10.5 & + & Single & ST & $\begin{array}{c}\text { SIQ3 }_{3} T_{3} T_{a v F} \\
T \downarrow V_{I}-V_{3}\end{array}$ \\
\hline 5 & 6 & II & 16 & 17 & 38 & 29 & $8 r$ & $8 \cdot 2$ & + & Single & ST & $\begin{array}{c}\mathrm{SIQ}_{3} \mathrm{~T}_{3} \mathrm{TavF} \\
\mathrm{T} \downarrow \mathrm{V}_{\mathrm{I}}-\mathrm{V}_{3}\end{array}$ \\
\hline 6 & 6 & 17 & $' v '=25$ & 13 & 37 & 22 & 85 & II O & - & Single & AF & RSR'VI $^{\prime}$ \\
\hline 7 & 6 & 7 & 9 & 9 & 35 & 27 & 85 & $8 \cdot 3$ & + & Single & ST & Normal \\
\hline 8 & 8 & 6 & 7 & 7 & 35 & 27 & - & - & - & Normal & NS & Normal \\
\hline 9 & 9 & Io & 15 & 12 & 37 & 22 & 97 & $8 \cdot 8$ & + & $?$ & ST & SIT $_{3}$ TavF \\
\hline 10 & 15 & 8 & IO & I3 & 33 & 26 & 91 & $8 \cdot 4$ & + & Wide & ST & $\begin{array}{l}\mathrm{SI}_{3} \mathrm{~T}_{3} \mathrm{TavF} \\
\mathrm{T} \downarrow \mathrm{V}_{1}-\mathrm{V}_{4}\end{array}$ \\
\hline II & 18 & Io & 14 & 13 & 35 & 24 & 89 & $6 \cdot 5$ & + & Single & ST & $\mathrm{SIQ}_{3} \mathrm{~T}_{3} \mathrm{TavF}$ \\
\hline 12 & 18 & 14 & 16 & 16 & 40 & 32 & 86 & $6 \cdot 5$ & + & Single & ST & RSR'VI $^{\prime}$ \\
\hline 13 & 24 & 16 & 18 & 17 & 45 & 32 & 53 & $6 \cdot 4$ & + & & ST & $\mathbf{R S R}^{\prime} \mathrm{VI}_{\mathbf{I}}$ \\
\hline 14 & 24 & I4 & 15 & 17 & 42 & 27 & 82 & $6 \cdot 5$ & + & Single & ST & Inf. myoc. infarct. \\
\hline 15 & 24 & 3 & 4 & 6 & 37 & 27 & 97 & $6 \cdot 2$ & - & $?$ & ST & $\begin{array}{c}\text { SIQ3 }_{3} T_{\text {TavF }} \\
\text { T } \downarrow V_{I}-V_{3}\end{array}$ \\
\hline 16 & 24 & I8 & 23 & $2 \mathrm{I}$ & 57 & 35 & 60 & $6 \cdot 8$ & + & Wide & NS & $\mathrm{SIQ}_{3} \mathrm{~T}_{3} \mathrm{TavF}$ \\
\hline 17 & 24 & I3 & 16 & 16 & 35 & 28 & 86 & $7 \cdot 6$ & + & Wide & ST & RBBB \\
\hline 18 & 36 & 18 & 12 & 12 & 38 & 26 & 98 & $6 \cdot 5$ & + & $?$ & ST & $\mathrm{Q}_{3} \mathrm{~T}_{3} \mathrm{~T} \downarrow \mathrm{VI}-\mathrm{V}_{4}$ \\
\hline 19 & 36 & 4 & 8 & 7 & 35 & 23 & 94 & $8 \cdot 2$ & + & Single & ST & RSR'VI \\
\hline 20 & 48 & 5 & 6 & 8 & 38 & 24 & 90 & $7 \cdot 7$ & - & Wide & NS & RBBB \\
\hline 21 & 48 & 0 & 2 & 0 & 30 & 22 & 86 & $9 \cdot 0$ & + & Single & ST & $\mathrm{SIQ}_{3} \mathrm{~T}_{3}$ \\
\hline 22 & 48 & 3 & 5 & 5 & 26 & 15 & 91 & $7 \cdot 2$ & - & Wide & NS & Normal \\
\hline \multirow[t]{2}{*}{23} & 48 & 7 & 9 & 7 & 37 & 24 & 96 & $8 \cdot 5$ & - & $?$ & ST & Normal \\
\hline & $\begin{array}{l}\text { Mean } \\
\text { SD } \pm\end{array}$ & $\begin{array}{l}9 \cdot 2 \\
4 \cdot 7\end{array}$ & & $\begin{array}{r}11 \cdot 5 \\
4 \cdot 9\end{array}$ & $\begin{array}{r}38.4 \\
6.8\end{array}$ & $\begin{array}{r}26 \cdot 6 \\
4 \cdot 7\end{array}$ & $\begin{array}{l}86 \cdot 4 \\
11 \cdot 2\end{array}$ & $\begin{array}{l}8 \cdot I \\
I \cdot 8\end{array}$ & & & & \\
\hline
\end{tabular}

* ST, sinus tachycardia; AF, atrial fibrillation; NS, normal sinus ; RBBB, right bundle-branch block. 


\section{(b) Physical signs}

Physical examination usually revealed an ill patient, dyspnoeic but not orthopnoeic, and sometimes centrally cyanosed. The arterial pulse was usually sharp and of small volume. Arterial hypotension (systolic pressure $<80$ $\mathrm{mm} . \mathrm{Hg}$ ) was seldom present in this series of patients, many of whom had been given vasopressor agents by the referring hospital. Tachycardia, sinus in all but the patient who had mitral stenosis and atrial fibrillation, was almost invariable in patients seen soon after the acute event ( 15 of 17 ) but present in only half of the patients seen at more than 24 hours. Signs of right ventricular failure (raised jugular venous pressure, gallop rhythm at the left sternal edge) were similarly influenced by the duration of embolism: of those patients seen within 24 hours all but 3 had raised jugular venous pressure and all but 2 (one of whom had atrial fibrillation) had a gallop rhythm. In contrast, of the 6 patients seen more than 24 hours after embolism only one had a raised jugular venous pressure and 3 of the 6 a gallop rhythm. Reliable observations of the nature of the second heart sound were made in 18 patients. A single second heart sound was the most common finding in patients seen within 24 hours (10 of $14,72 \%$ ) but wide splitting varying with respiration was observed both in those seen at more than 24 hours and, infrequently, in those seen earlier. The pulmonary component was never louder than the aortic component. Right ventricular hypertrophy was never present on palpation, but brief, non-sustained movements coincident with the first and second heart sounds could occasionally be palpated at the left sternal edge.

\section{(c) Electrocardiogram}

The electrocardiogram usually showed abnormalities of conduction or repolarization of the right ventricle (in $78 \%$ of patients). Thus in 5 patients an $\mathrm{SrQ}_{3} \mathrm{~T}_{3}+\mathrm{T}$ wave inversion $\mathrm{V}_{\mathrm{I}}-\mathrm{V}_{4}$ pattern was seen. In 5 others complete right bundle-branch block was present while 4 patients had an RSR' pattern in VI. Four patients had $\mathrm{SIQ}_{3} \mathrm{~T}_{3}$ TavF pattern. Four patients $(17 \%)$ had a normal electrocardiogram while the patient who had an inferior myocardial infarct previously retained an unchanged cardiogram in spite of massive pulmonary embolism.

\section{(d) Chest radiograph}

Chest radiographs were available for review in 22 of the 23 patients and showed altera- tions in pulmonary vasculature in almost all patients. Thus oligaemia of some part of the lung fields corresponding to the angiographically shown distribution of embolism was seen in all but one patient, who had a severe dorsal kyphoscoliosis making impossible the interpretation of vascular changes in the plain radiograph. Dilatation of the main pulmonary trunk was seen in only 3 patients, one of whom had mitral stenosis in addition to recent embolism. Unilateral elevation of the diaphragm was present in 7 patients and shadows consistent with pulmonary infarcts in II. Enlarged, plump hilar shadows were seen in ro patients.

(2) Pulmonary arteriograms Pulmonary arteriograms showed involvement of at least half of the major pulmonary arteries in all patients. Filling defects with convex borders, typical of recent embolism (Fig.), were seen in all patients, commonly at the bifurcation of the major pulmonary arteries. Wormlike filling defects outlined in the smaller vessels by a rim of contrast medium were also seen. In many instances the filling defects totally occluded the vessels so that there was no peripheral filling. In other instances the vessels were only partially occluded permit-

FIG. Case 13 showing convex bulging of filling defect into contrast-filled right pulmonary artery due to embolus at bifurcation of right pulmonary artery. There are also filling defects due to emboli in the left pulmonary artery.

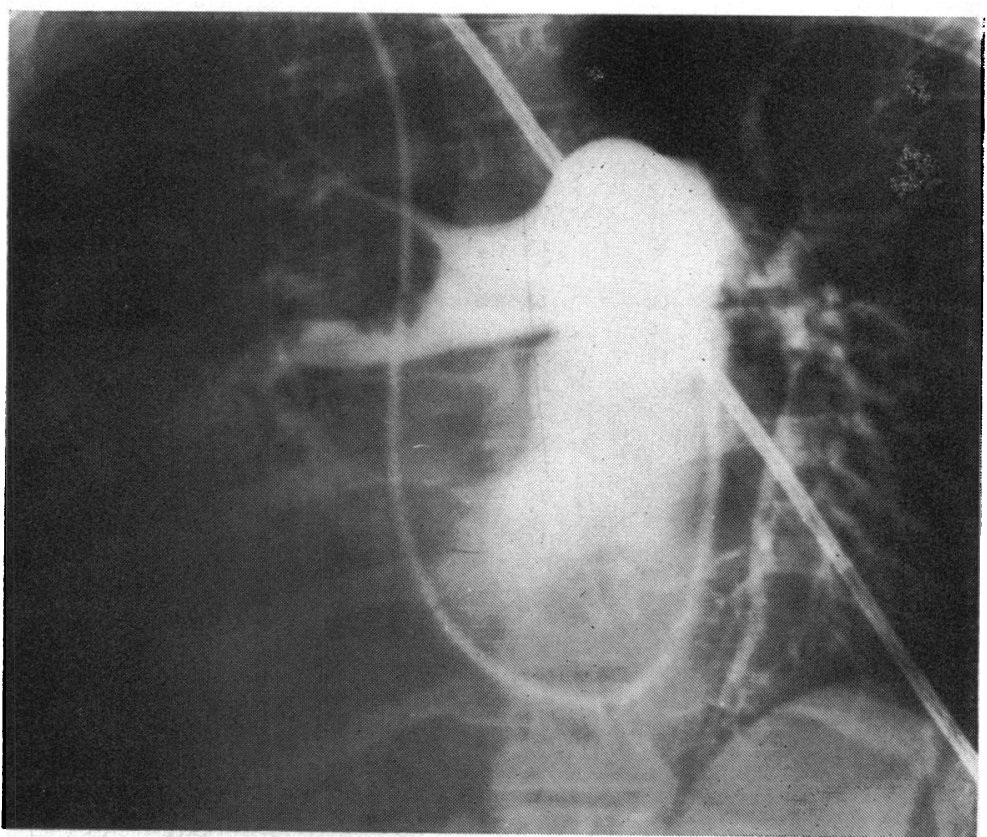


ting distal filling, though the rate of distal filling by contrast medium was often slower than in unaffected areas. The distribution of pulmonary arterial filling was parallelled by pulmonary venous filling which was reduced or absent in veins draining affected areas.

(3) Haemodynamic findings In all patients there was an only moderate increase in pulmonary artery systolic (average $38 \cdot 4 \pm 6 \cdot 8$ $\mathrm{mm}$. $\mathrm{Hg}$ ) and mean (average $26 \cdot 6 \pm 4 \cdot 7 \mathrm{~mm}$. $\mathrm{Hg}$ ) pressures. Right ventricular end-diastolic (RVED) pressures were $12 \mathrm{~mm}$. $\mathrm{Hg}$ or above in 14 of the 23 patients (average $11.5 \pm$ $4.9 \mathrm{~mm}$. $\mathrm{Hg}$ ), with a commensurate rise in right atrial pressure. For patients studied 24 hours or less after the acute event, the values for mean right atrial pressure and RVED $(10.8+4 . \mathrm{I}$ and $13.3 \pm 3.9 \mathrm{~mm}$. $\mathrm{Hg}$, respectively) were significantly $(\mathrm{p}<0.005)$ higher than these values in patients studied at more than 24 hours $(4.5 \pm 2.9$ and $6.5 \pm 3.9 \mathrm{~mm}$. $\mathrm{Hg}$, respectively). Arterial oxygen desaturation $(<95 \%)$ was present in all but 4 of the patients (average $86.4 \pm I I \cdot 2 \%$ ). In 2 patients (Cases 13 and 16 ) arterial oxygen desaturation was obvious ( $53 \%$ and $60 \%$ ). In all patients arteriovenous (AV) oxygen differences were abnormally wide (average $8 \cdot \mathrm{I} \pm \mathrm{I} \cdot 8 \mathrm{ml}$./100 ml.). The cardiac index was abnormally low in every patient and below $2 \cdot 01 . / \mathrm{min} . / \mathrm{sq} . \mathrm{m}$. in 21 of the 23 patients.

\section{Discussion}

Pulmonary embolism is responsible for some 2-3000 deaths/year in the United Kingdom alone (General Register Office, 1968) and its incidence may be increasing (Morrell, Truelove, and Barr, 1963); though many patients die before any effective treatment is possible, there remains a proportion who survive long enough for investigation and treatment to be instituted (Gorham, 196r ; Donaldson et al., 1963). The patients reported here represent just such patients who have survived massive pulmonary embolism long enough to be transferred to this hospital. The findings reported relate, therefore, to the effects of massive pulmonary embolism 2-48 hours after the acute event and not to the immediate sequelae. The patients are also a selected group in that only two had pre-existing cardiorespiratory disease; thus we are able to describe the haemodynamic consequences, and hence the clinical findings of acute right ventricular outflow obstruction in man as an isolated disturbance.

There are few published data reporting the haemodynamic findings in massive pulmonary embolism close to the acute incident. Previous reports (Del Guercio et al., 1966; Leland and Sasahara, 1965; Sasahara et al., 1966) of the haemodynamic consequences of pulmonary embolism have shown a wide variation in findings between patients; such variation could be attributed to the inclusion of patients with pulmonary embolism of differing severity and duration as well as the inclusion of patients with pre-existing cardiorespiratory disease. Thus 63 per cent of patients reported by Sasahara et al. (1966) had previous heart disease, the majority having rheumatic mitral valve disease. Both very high and normal pulmonary artery pressures were recorded, in contrast to our findings which were strikingly uniform in relation to the pulmonary arterial pressure, which was only moderately raised in all patients. Recently, Hirsh et al. (1968) have reported a series of 18 patients of whom 6 had massive embolism without previous cardiorespiratory disease; this latter group of patients had pulmonary artery pressures closely similar to those reported here. It appears that the acutely stressed right ventricle is unable to generate a pressure adequate to overcome the increased resistance to ejection; the cardiac output falls and right ventricular failure is manifested by a rise in enddiastolic pressure. These findings are confirmed at the time of embolectomy for massive embolism when a dilated poorly contracting right ventricle is an invariable finding ( $M$. Paneth, 1970, personal communication).

Clinically low cardiac output is reflected in arterial hypotension, a small volume jerky pulse, and a constricted peripheral vasculature. Historically the acute reduction in cardiac output may result in 'collapse' or circulatory arrest due to impaired cerebral or coronary blood flow, while the occasional complaint of central chest pain resembling angina may have a similar mechanism.

Similarly, right ventricular failure is manifested clinically by an increase in jugular venous pressure and a gallop rhythm at the left sternal edge. The mechanism of this gallop rhythm may depend on a powerful right atrial contraction (S4), an abnormal ventricular filling sound $\left(\mathrm{S}_{3}\right)$, or summation of both factors in the presence of a tachycardia. Thus the patient in established atrial fibrillation did not have a gallop and two patients with transient atrial fibrillation lost their gallop rhythm during these episodes. Similarly, of the 5 patients in sinus rhythm who did not have a gallop, all had a normal right ventricular end-diastolic pressure and 4 of these did not have a sinus tachycardia.

Palpation did not reveal right ventricular 
hypertrophy in any patient; this is not surprising in view of the operative finding of a dilated but poorly contracting right ventricle. Delayed pulmonary valve closure $\left(\mathbf{P}_{2}\right)$ might be expected to result from a poorly contracting right ventricle with delayed ejection; thus wide splitting of the second heart sound in pulmonary embolism has been reported (Cobbs, Logue, and Dorney, 1966) and was observed occasionally in our patients. In our experience, however, the second heart sound was more usually single, loud, and palpable.

Conspicuous enlargement of the pulmonary artery on the plain chest radiograph was not a feature in this group of patients, as might be expected when there is only moderate pulmonary hypertension and a reduced pulmonary blood flow. Some enlargement of the pulmonary artery could, however, be detected on the arteriograms, particularly when, after surgical or thrombolytic therapy, a repeat arteriogram showed reduction in pulmonary artery diameter. First examination of portable plain chest radiographs did not appear to be helpful in the diagnosis of massive pulmonary embolism (Sutton et al., 1969); however, in retrospect these films did show oligaemia of the affected zones and we now recognize this as a valuable aid to diagnosis in massive pulmonary embolism (Kerr, Simon, and Sutton, to be published).

The haemodynamic consequences of pulmonary embolism must be determined by the duration and severity of the disease as well as the presence or absence of complicating cardiorespiratory disease. As far as could be determined, all the patients in this series had embolism of approximately equal severity; duration of the disease is difficult to determine exactly from the history which may have included minor premonitory episodes of pulmonary infarction. However, with one exception, patients studied more than 24 hours after the acute event differed from the remainder in that there was no longer evidence of right ventricular failure. While these findings may represent some spontaneous resolution, pulmonary arteriograms still showed massive embolism; an alternative explanation could be that adaptation of the right ventricle to the stress has occurred. Such patients may present diagnostic difficulty in terms of severity. A second group of patients presenting diagnostic difficulty are those in whom minor embolism has occurred in the presence of existing cardiorespiratory disease, including patients with chronic pulmonary thromboembolism who have suffered a recent minor embolism. In such patients haemodynamic and clinical find- ings may exactly mimic massive embolism. Uncomplicated minor embolism in our experience is not accompanied by haemodynamic disturbance. Because of such diagnostic difficulties and in order to assess results of treatment, it is our practice to perform pulmonary arteriograms on all patients with a presumptive diagnosis of massive pulmonary embolism. While arteriography may be hazardous in such acutely ill patients, Scannell (1967) and Sautter (1965) report 25 and 35 cases, respectively, of acute massive pulmonary embolism studied by arteriography without mortality. Of the 23 patients with massive embolism reported here none suffered permanent ill effects from arteriography, but several experienced a profound fall in systemic arterial pressure immediately after arteriography and 3 required a brief period of external cardiac massage. This brief hypotensive period was not associated with electrocardiographic abnormalities and was presumably due to the vasodilator effects of contrast medium in patients with a cardiac output fixed at a critically low level; such a profound hypotensive response to vasodilatation again suggests that the acutely stressed right ventricle is unable to increase the pulmonary artery pressure to a degree adequate to maintain pulmonary and, therefore, systemic flow.

\section{References}

Cobbs, B. W., Logue, R. B., and Dorney, E. R. (1966). The second heart sound in pulmonary embolism and pulmonary hypertension. American Heart fournal, 71, 843.

Del Guercio, L. R. M., Cohn, J. D., Feins, N. R., Coomaraswamy, R. P., and Mantle, L. (I966). Pulmonary embolism shock. Physiologic basis of a bedside screening test. Fournal of the American Medical Association, 196, 751.

Donaldson, G. A. Williams, C., Scannell, J. G., and Shaw, R. S. (1963). A reappraisal of the application of the Trendelenburg operation to massive fatal embolism. New England fournal of Medicine, 268, 171.

Du Bois, D., and Du Bois, E. F. (1916). Clinical calorimetry: a formula to estimate the approximate surface area if height and weight be known. Archives of Internal Medicine, 17, 863.

General Register Office (1968). The Registrar General's Statistical Review of England and Wales (1967). Part I. Tables, Medical, p. 26. H.M.S.O., London.

Gorham, L. W. (196I). A study of pulmonary embolism. Archives of Internal Medicine, 108, 8.

Hirsh, J., Hale, G. S., McDonald, I. G., McCarthy, R. A., and Pitt, A. (1968). Streptokinase therapy in acute major pulmonary embolism: effectiveness and problems. British Medical fournal, 4, 729.

Kerr, I. H., Simon, G., and Sutton, G. C. To be published.

Leland, O. S., and Sasahara, A. A. (1965). Hemodynamic observations in patients with pulmonary thromboembolism. In Pulmonary Embolic Disease, p. I IO. Ed. by A. A. Sasahara and M. Stein. Grune and Stratton, New York. 
Morrell, M. T., Truelove, S. C., and Barr, A. (I963). Pulmonary embolism. British Medical fournal, 2, 830.

Robertson, J. D., and Reid, D. D. (1952). Standards for the basal metabolism of normal people in Britain. Lancet, 1, 940.

Sasahara, A. A., Sidd, J. J., Tremblay, G., and Leland, O. S. (1966). Cardiopulmonary consequences of acute pulmonary embolic disease. Progress in Cardiovascular Disease, 9, 259.

Sautter, R. D. (1965). Massive pulmonary thromboembolism: experience with 12 pulmonary embolectomies. Fournal of the American Medical Association, 194, 336.
Scannell, J. G. (1967). The surgical management of acute massive pulmonary embolism. Progress in Cardiovascular Disease, 9, 488.

Scholander, P. F. (1947). Analyzer for accurate estimation of respiratory gases in one-half cubic centimeter samples. Fournal of Biological Chemistry, 167, 235.

Sutton, G. C., Honey, M., and Gibson, R. V. (1969). Clinical diagnosis of acute massive pulmonary embolism. Lancet, I, 271.

Zijlstra, W. G., and Mook, G. A. (1962). Medical Reflection Photometry. Van Gorcum, Assen, Netherlands. 